\title{
Longitudinal Models for Project Expenditure Plans
}

\author{
T. Weir and K. Johns
}

\author{
Joint and Operations Analysis Division, Defence Science and Technology Group, Department of \\ Defence \\ Email: terence.weir@dst.defence.gov.au
}

\begin{abstract}
The ability of project managers to generate accurate budget predictions for capital investments is critical for successful project completion and the management of the Australian Department of Defence budget. Accordingly, this paper discusses non-linear regression models to analyse planned time-phasing of expenditures for projects in an Australian Defence capital investment program. Earlier research on time-phasing primarily focuses on the theoretical foundation for applying the cumulative distribution function, usually of functional forms such as Rayleigh and Weibull curves, to model the distribution of project expenditures. Previous approaches have also relied on elementary regression techniques to estimate the parameters of the curves. However, these analyses have generally ignored the longitudinal, repeated measures nature of the data for each project, potentially compromising results because the data within projects is correlated. Here, a non-linear mixed-effects model is used to estimate the parameters of the time-phasing distributions for a large number of major capital investment projects. Unlike ordinary linear or non-linear regression, this has the advantage of accounting for within project correlation and unequal variance. The model can be thought of as a hierarchical model involving both fixed-effects associated with the population of projects and randomeffects accounting for unexplained inter- and intra-project variability. The fixed part of the model incorporates military domain effects and project epochs, and random effects account for heteroscedasticity and correlations between the repeated measures. Analysis reveals significant differences between project's time-phasing due to their domain and epoch. The model may be used by project and portfolio planners to estimate required spending plans against project schedule and total planned expenditure; it therefore provides a tool to measure project and financial risk.
\end{abstract}

Keywords: $\quad$ Non-linear regression, capital investment, time-phasing 


\section{INTRODUCTION}

A significant proportion of Department of Defence (Defence) expenditure is for the Approved Major Capital Investment Program (AMCIP). In recent years Defence has budgeted approximately $\$ 4500 \mathrm{~m}$ annually for expenditure on major projects (Department of Defence, 2015). Expenditure will increase significantly in the future as Defence implements an expanded capability portfolio (Department of Defence, 2016).

Modern systems engineering literature suggests that greater efforts in planning and designing systems help reduce rework and cost over-runs later in programs (see e.g. Blanchard, 2008). Less effort early on in projects can lead to poor understanding of user requirements, scope creep and unrealistic estimates of cost and time to completion. Other published research shows that funding provided in the wrong fiscal year of development may result in productivity inefficiencies, schedule slips, and increased program costs (Unger et.al., 2004). For these reasons, a model for the spread of funding across a project's life would aid analysts and managers. Furthermore, in response to the First Principles Review (Peever et al., 2015), Defence has sought to improve its capability in parametric modelling of projects' costs, schedule and risk. Peever et al. also recommends Defence cease expenditure slippage and portfolio overprogramming (planning for expenditure slippage) as management tools used on project and portfolio expenditure plans. Defence has a long history of using overprogramming and it will require improved project planning to avoid expenditure variations. The research here provides a quantitative methodology for project planning in Defence. It makes a direct contribution to Defence's analytic capability by developing a method of parameterising project planned expenditures and relating the parameters to project characteristics.

An examination of year-on-year expenditure patterns in AMCIP projects was undertaken by Weir (2017). That analysis employed a log-normal linear mixed model to regress actual expenditure against planned expenditure where (project) time was a covariant. Another approach to model project expenditure, time-phasing, has been investigated by several other authors, although in other contexts; see e.g. Burgess (2006) and Brown et al. (2015). Time-phased modelling of expenditure plans provides a statistically robust method of analysis, especially for project planning. This applies the cumulative distribution function, commonly referred to as an S-curve, to project spend spreads. Some earlier studies have made use of the single parameter Rayleigh distribution (see e.g. Abernethy, 1984), but later studies, including those of Burgess and Brown et al. have used the multi-parameter Weibull distribution because it enables more flexibility and precision. It is noted that these analyses assume independence of observations and do not account for correlated data. These assumptions may not be valid because the expenditures come from repeated observations on the same project.

This paper models project expenditures for AMCIP projects in the period 1996/97 to 2012/13 using a nonlinear mixed effects model (NLMM) to describe the trajectory of costs in each project. Planned and actual expenditure in each AMCIP project in each year of its duration has been recorded by Defence for financial planning and reporting and provides the data set for analysis. The project financial data is accompanied by, inter alia, organisational data that identifies the domain of the investment, such as land, sea or air. Because variations from expenditure plans have the potential to create cash flow problems in Defence, developing a model that explains the time-phasing of expenditure will improve project planning and portfolio financial management. From a Defence portfolio management perspective, it is also desirable to understand if families of projects behave differently from each other because the differences may be due to varying management practices across project domains or to the nature of domains. In the following sections of this paper we describe the project expenditure plans. We give a brief overview of the data and previous work. We then estimate the Weibull parameters for each project and relate the parameters, where possible, to characteristics of projects.

\section{DATA FORM AND SCOPE}

The data set analysed here consists of 196 projects with 918 observations of planned expenditure. Each project has a minimum of four observations (plus the origin, making a minimum of five observations for modelling). Any missing data is considered missing at random because the data is missing both on the covariate of time and (the outcome) expenditure. Expenditures have been adjusted for inflation. Each project belongs to one of five domains - Air (AIR), Joint (JNT), Land (LND), Other (OTH) and Sea (SEA) and to one of three epochs - projects commenced prior to 1997 (Epoch_1), projects commenced between 1997 and 2005 (Epoch0) and projects commenced after 2005 (Epoch1). 
Observations have been taken only from years one to 10 of a project's duration ${ }^{1}$. A histogram of the distribution of project lengths is at Figure 1(A).

A

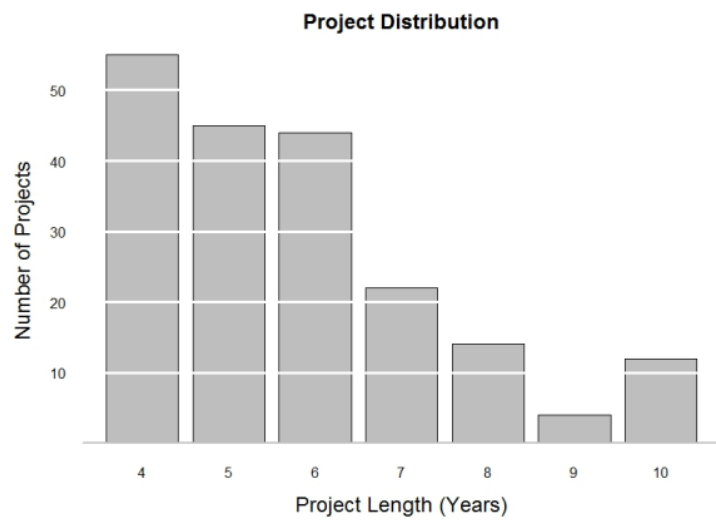

B

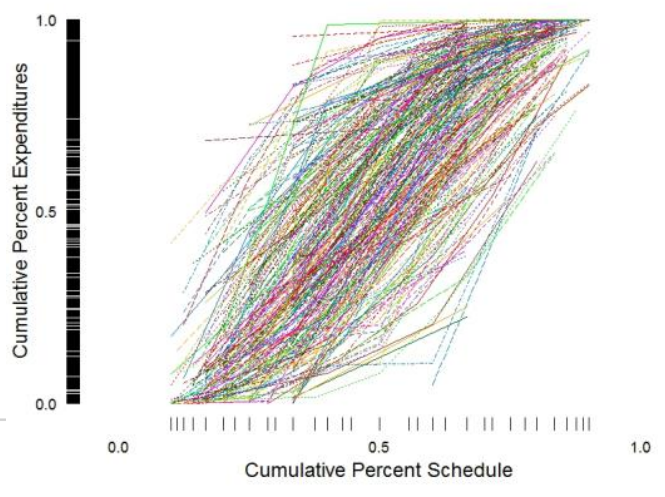

Figure 1. (A) Distribution of projects by their length (B) planned cumulative percent expenditure versus percent schedule for all projects with marginal distribution of each variable

Each project's planned expenditures are converted to a percent cumulative expenditure by percent schedule. Profile plots for cumulative percent expenditure versus percent schedule for all projects are shown in Figure 1(B). There is clearly considerable variation of expenditure profiles between projects.

A

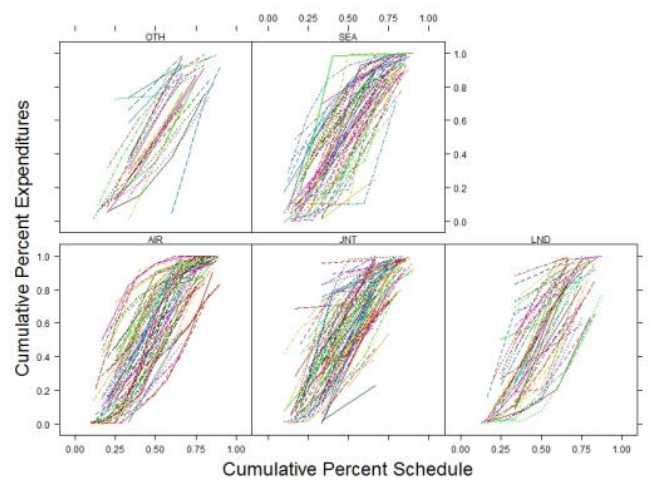

B

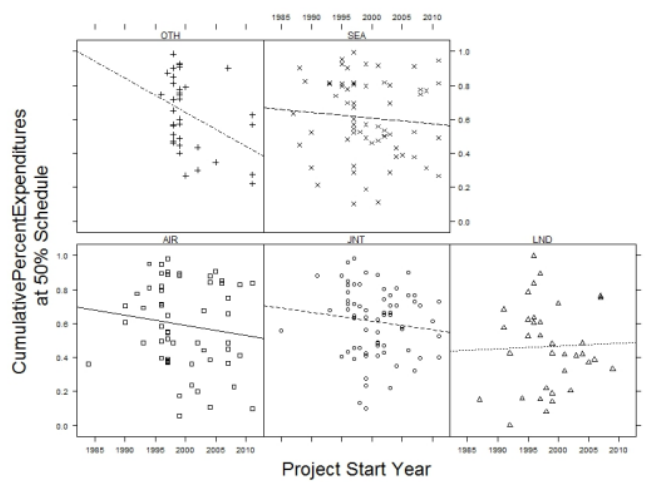

Figure 2. (A) Plot for the planned cumulative $\%$ expenditure versus $\%$ schedule for projects in each Domain (B) Percent expenditures at $50 \%$ schedule by project start year and Domain

The range of percentage expenditures at the development midpoint is substantial. A mean of $58 \%$ of total expenditures occurs at the $50 \%$ development point. There appears little difference in profiles even when domain is considered at Figure 2(A). After aggregating we find that mean expenditure at the 50\% development point by domain is as follows: AIR 59\%, JNT 61\%, LND 47\%, OTH 63\% and SEA $61 \%$. So overall spending considerably leads development in all but one of the domains. It is worth noting that the percentage spend reflected in these projects is considerably more than that of comparable studies at Brown et al. (2015) but similar to that in Burgess (2006). Figure 2(B) shows that expenditures by the development midpoint have generally decreased over time. This may reflect an increasingly conservative risk based acquisition process fostered by successive reviews. It is noted that "OTH" projects have been phased out in recent years, and replaced by "JNT" projects.

\section{PARAMETERISING PROJECT DATA}

Assuming a nonlinear evolution, and denoting by $\mathrm{t} \geq 0$ the time, the accumulation of project expenditures can be modelled by the following Weibull Cumulative Distribution Function (CDF) ${ }^{2}$

\footnotetext{
${ }^{1}$ Some projects extend beyond 10 years in their execution, but the original plan is constrained to 10 years in AMCIP
} 


$$
F(t)=1-\exp \left[-\left(\frac{t}{\beta}\right)^{\alpha}\right]
$$

where $\alpha>0$ and $\beta>0$ represent respectively the shape parameter and the scale parameter. The scale parameter $\beta$ determines the steepness of the CDF and the shape parameter $\alpha$ allows proportions to evolve at other than linear rates.

The plot at Figure 1(B) gives an overall view of the structure of the data. We constrain the analysis to the Weibull distribution as some preliminary background analysis suggests it provides better a fit than complementary distributions of Beta and Raleigh. We commence by estimating parameters for a simple model of the data that gives the mean growth assuming independent observations.

The parameters may be estimated by fitting

$$
y_{i j}=\frac{\left(1-\exp \left[-\left(\frac{t_{j}}{\beta}\right)^{\alpha}\right]\right)}{\left(1-\exp \left[-\left(\frac{1}{\beta}\right)^{\alpha}\right]\right)}
$$

to the data where $y_{i j}$ is cumulative expenditure in project $i$ at time $t_{j}$. The denominator in (2) is required to account for the infinite tail of the Weibull density.

We first use use nonlinear least squares estimation to calculate the parameters of (2) assuming uncorrelated errors. Box plots of the residuals for each project show some projects having all residuals larger than others, suggesting that there are project-specific components affecting the response. This assessment is supported by a plot of the confidence intervals for the parameters. This variability may be modeled by random effects on the parameters of the Weibull distribution. Reducing correlations for planned expenditure over time suggests that an appropriate model may require an autoregressive variance structure. Plots of the shape and scale parameters show some weak evidence for a difference in each parameter across Domains and Epoch. So Domain and Epoch may be useful as a covariant of the shape and scale parameters in (2).

A nonlinear least squares (NLLS) fit of (2) on the data using Epoch as a covariant of the beta parameter is displayed at Figure 3. Although the plots of the modelled fits appear to fit the data well, an analysis

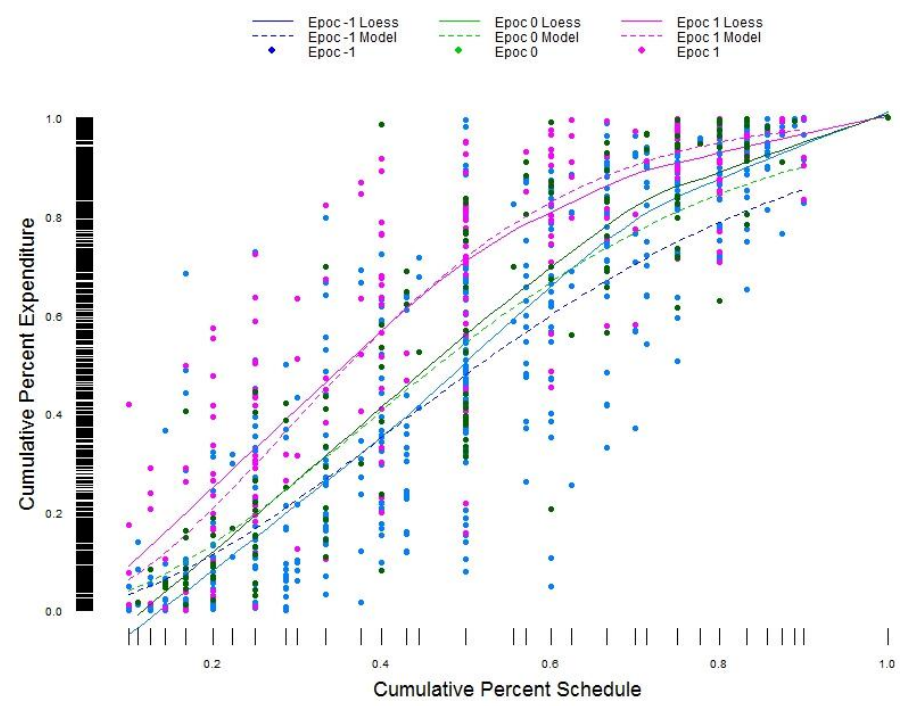

Figure 3. Loess and modelled nonlinear least squares fits to the data with marginal distributions of the variables

of the residuals does not support the assumptions of a NLLS model. Again, it is expected that incorporating random effects and a variance structure will produce a better fit to the data.

Previous S-curve research, has hypothesised that project length, project total expenditure and domain are important predictor variables for the Weibull parameters (see eg. Brown et al., 2002; Burgess, 2006). Changes in Defence capability management (see e.g. Kinnaird et.al., 2003) mean that project

\footnotetext{
${ }^{2}$ Often referred simply as distribution
} 
epoch may also be an important factor. Preliminary modelling investigations showed that only domain and epoch were applicable predictor variables for this study.

\section{NONLINEAR MIXED EFFECTS MODELS}

Mixed models differ from more familiar linear models (e.g. linear regression, ANOVA) through their ability to cope with correlated data and unequal variances. Mixed models are very useful to analyze data with a hierarchical structure, where measures from distinct units or subjects are independent and those from within subjects are correlated. Essentially, mixed models segment data into two parts: fixed effects and random effects. The expected values of the observations are described by the fixed effects, and the variance and covariance of the observations are described by the random effects. In particular, mixed models are appropriate to analyze repeated measures in longitudinal studies where each subject of the study is measured multiple times. Mixed effects models give correct estimates of treatment and other fixed effects in the presence of the correlated errors that arise from a data hierarchy.

NLMM extend linear regression models in two ways - firstly to incorporate a nonlinear response to a predictor variable and secondly to allow the predictor variable to be modeled as mixed effects linear model. Nonlinear regression methods are suited for analysing data for which there is an empirically or theoretically established functional relationship between response and predictor. NLMM for repeated measures can be thought of as a hierarchical model involving both fixed-effects associated with the population parameters and random-effects accounting for unexplained inter- and intra-individual variability. At the first-stage model, the intra-individual (residual) variability describing the difference between the individual predicted values and the observations is modelled as

$$
y_{i j}=f\left(\phi_{i}, v_{i j}\right)+e_{i j}, i=1, \ldots, N, j=1, \ldots N_{i}
$$

where $y_{i j}$ is the $\mathrm{j}$-th response for the $\mathrm{i}$-th unit, $\phi_{i}$ is a subject specific parameter vector and $v_{i j}$ is a vector of covariates. The error terms $e_{i j}$ are assumed independently and identically distributed normal random variables with mean zero and variance $\sigma^{2}$.

At the second-stage of the hierarchy, the model relates the parameters of the different individuals using a linear mixed model,

$$
\phi_{i}=X_{i j} \beta+Z_{i j} b_{i}
$$

where $X_{i j}$ and $Z_{i j}$ are design matrices for the fixed-effects vector $\beta$ and random-effects vector $b_{i}$, respectively. The inter-individual variability is modelled by the random-effects vector $b_{i}$ which consists of $p$ zero-mean variables assumed to be independent and identically distributed (traditionally thought to be the multivariate normal distribution) with variance-covariance matrix $\mathbf{G}$. The residual error terms $e_{i j}$ and the random effects $b_{i}$ are assumed normally distributed and independent for all $i$ and $j$.

\section{MODEL FITTING}

Here we consider a NLMM model that includes random effects to account for possible positive withinsubjects correlations. The NLMM, with random intercepts, may be expressed as in the form of (4) via the function described at (2), and

$$
\begin{gathered}
\alpha_{i k l}=\rho+u_{i}+\varepsilon_{k} \text { Epoch }_{k}+\delta_{l} \text { Dom }_{l} \\
\beta_{i k l}=\eta+v_{i}+\tau_{k} \text { Epoch }_{k}+\omega_{l} \text { Dom }_{l}
\end{gathered}
$$

for the shape and scale parameters respectively, where $\rho$ and $\eta$ are the mean values of shape and scale parameters respectively and $u_{i} \sim N\left(0, \sigma_{b_{\alpha}}^{2}\right)$ and $v_{i} \sim N\left(0, \sigma_{b_{\beta}}^{2}\right)$ are independent random variables corresponding to the random effects of project $i$. Epoch $h_{k}$ and $D_{o m}$ are respectively indicator variables for the Epoch and Domain of each project with corresponding parameters $\varepsilon_{k}, \delta_{l}, \tau_{k}, \omega_{l}$ where 
$k$ and $l$ range over the levels of Epoch and Domain respectively. The errors $e_{i j}$ in (4) are independent and follow $N\left(0, R_{i}\right)$ distributions, where, as suggested by the preliminary analysis, each $R_{i}$ has different variances for each time period of project $i$ and incorporates an autoregressive covariance structure over time to model the correlation of expenditures within project $i$. It is expected that $\operatorname{cor}\left(u_{i}\right.$, $v_{i}$ ) is zero. The model was fitted via maximum likelihood (with restricted maximum likelihood for the within-subjects covariance parameters) using methods implemented in the nlme library (Pinheiro et.al., 2013) in $R$.

A summary of the model is given at Table 1. The fixed effects are significant. The estimated covariances (not reported here) between the fixed effects are very small indicating that the parameters are all identifiable. Some parameter intervals for the variance function (different variance per time period) include unity, but there is sufficient evidence (L-Ratio 61.0730 on 8 Dof, p-value $<0.0001$ ) to reject the assumption of homoscedasticity. Autocorrelation in the covariance structure is supported by a likelihood ratio test (L-Ratio 64.5478 on 1 Dof, p-value < 0.0001). Other likelihood tests found no improvement in fit assuming different variances per Epoch or Domain instead of time (year).

Table 1. Summary of the final model

\section{Fixed Effects}

\begin{tabular}{|c|c|c|c|c|c|c|c|}
\hline & $\alpha$. intercept & a.Epoch_1 & $\alpha$. Epoch0 & $\alpha . J N T$ & $\alpha . L N D$ & $\alpha . \mathrm{OTH}$ & $\alpha . S E A$ \\
\hline Value & 1.589 & 0.0723 & -0.238 & -0.137 & 0.186 & -0.144 & 0.009 \\
\hline Std.Error & 0.0868 & 0.0803 & 0.0934 & 0.0786 & 0.098 & 0.1217 & 0.078 \\
\hline \multirow[t]{2}{*}{ p-value } & $<.0001$ & 0.012 & 0.0743 & 0.0257 & 0.015 & 0.9404 & 0.606 \\
\hline & $\beta$.intercept & $\beta . E p o c h \_1$ & $\beta . E p o c h 0$ & $\beta . J N T$ & $\beta . L N D$ & $\beta . \mathrm{OTH}$ & $\beta . S E A$ \\
\hline Value & 0.71 & 0.0322 & -0.048 & 0.016 & 0.076 & 0.075 & 0.011 \\
\hline Std.Error & 0.02 & 0.0209 & 0.0252 & 0.022 & 0.025 & 0.035 & 0.021 \\
\hline p-value & $<.0001$ & 0.0003 & 0.0344 & 0.701 & 0.006 & 0.041 & 0.589 \\
\hline
\end{tabular}

\section{Random Effects}

\begin{tabular}{llll}
\hline & $\alpha . i n t e r c e p t$ & $\beta . i n t e r c e p t$ & Residual \\
StdDev & 0.38012 & 0.09581 & 0.04005
\end{tabular}

Variance Function Different

standard deviations per stratum

\begin{tabular}{llllllllll}
\hline Year & 1 & 2 & 3 & 4 & 5 & 6 & 7 & 8 & 9 \\
Var Par & 1 & 2.1451 & 1.7102 & 0.9968 & 0.893 & 0.8828 & 0.6049 & 0.5661 & 0.7418 \\
\hline
\end{tabular}

Autoregressive Correlation Parameter: 0.5686

Model: AIC: -2047.323 BIC: $-1922.374 \quad$ LogLik: 1049.661

The variance parameter estimates of a model including correlation between the random effects $u_{i}$ and $v_{i}$ indicate that the correlation is insignificant (L-Ratio 1.6853 on 1 Dof, p-value 0.1942). Diagnostics show that the model fit is adequate. There are some outliers, residuals larger, in absolute value, than the

97.5th percentile of the standard normal distribution but not in an unexpected number, and $25 \%$ of the outliers were generated by one project. Inclusion of the variance by stratum parameter and autocorrelation parameter removed any correlation in the residuals. Examination of the conditional residuals suggests mild deviations from the normality assumption, especially for smaller residuals, but this is unlikely to jeopardize the results. The variance is stable over Domains and Epochs. The 
Estimated Best Linear Unbiased Predictors (EBLUPs) of the random effects are approximately normally distributed.

Selections of the augmented predictions of the fitted model are shown at Figure 4 which show the marginal and project specific responses. The modelled project specific predictions closely follow the observations.

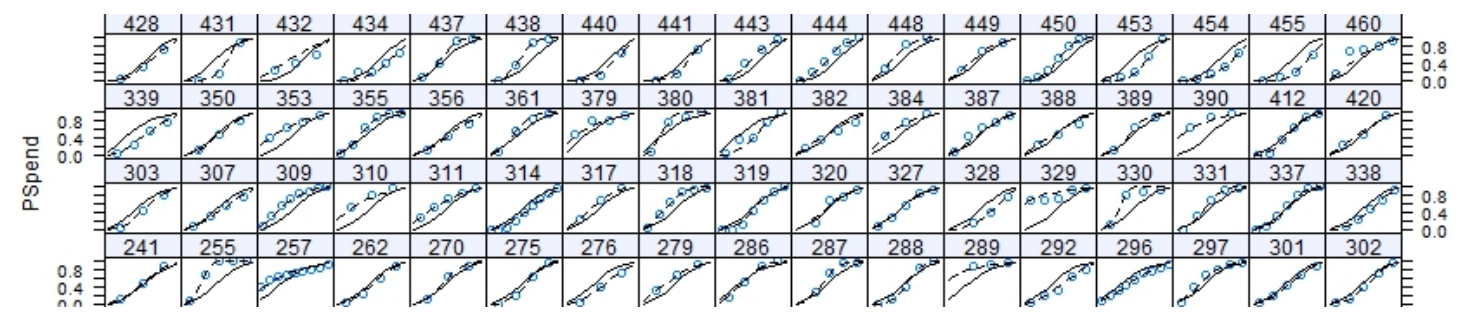

Figure 4. A selection of projects' observed and predicted percentage expenditures plotted vs. percentage time. Population (mean) predictions (solid line), individual project predictions (dashed line) and observed planned percentage expenditures (circles)

\section{CONCLUSIONS}

This report examined project expenditure plans for AMCIP projects in the period 1996/97 to 2012/13. The expenditure plans were modelled as a Weibull distribution using a NLMM. The NLMM allows for a detailed modelling of the within-projects correlation structure and the prediction of individual random effects. The modelled within project correlation structure reflects the observed autocorrelation of within project expenditures. The random effects are at the project level, and represented in the model as random intercepts of the Weibull shape and scale parameters. The modelling demonstrates that there are both Project Epoch and Project Domain effects on the shape and scale parameters, suggesting that project planning has changed over time and is different across domains. While there have been a number of reviews that have influenced project planning over the years, it is surprising that there remain differences in planning across domains because common processes and management arrangements are in place for the management of AMCIP projects. There are few outliers exhibited in the model and a significant proportion of those were due to one project. The model may be used by project and portfolio planners to test proposed spending plans against those typically seen in a long history of projects; hence the model provides a measure of project and financial risk.

\section{REFERENCES}

Abernethy, T.S., (1984). An application of the Rayleigh distribution to contract cost data, Naval Postgraduate School.

Brown, T.W., E.D White III, and M.A. Gallagher (2002). Weibull-based Forecasting of R\&D Programs, Journal of Cost Analysis and Management, 4, 41-51.

Brown, G.E., E.D. White III, J.D. Ritschel, and M.J. Seibel (2015). Time Phasing Aircraft R\&D Using the Weibull and Beta Distributions, Journal of Cost Analysis and Parametrics, 8:3, 150-164.

Burgess, E., (2006). R\&D Budget Profiles and Metrics, Journal of Parametrics, 25, 11-29.

Blanchard, B., (2008). Systems Engineering Management, New York: John Wiley \& Sons.

Department of Defence (2015). Defence Portfolio Budget Statements 2015-16

Department of Defence (2016). 2016 Integrated Investment Program.

Kinnaird, M., L. Early, and W. Schofield, (2003). Defence Procurement Review, Department of Defence, Canberra.

Peever, D., R. Hill, P. Leahy, J. McDowell, and L Tanner (2015). Creating One Defence - The First Principles Review of Defence, Department of Defence.

Pinheiro, J., D. Bates, S. DebRoy, D. Sarkar, and the R Development Core Team, (2013). nlme: Linear and Nonlinear Mixed Effects Models. R package version 3.1-108.

Unger, J. M., Gallagher, and E. White (2004). "R\&D Budget-driven Cost and Schedule Overruns," Journal of Cost Analysis and Management 6, 80-97.

Weir, T., (2017). Regression models for project expenditures, Lecture Notes in Management and Industrial Engineering, Data and Decision Sciences in Action, Ch 20, DOI: 10.1007/978-3-31955914-8_20. 